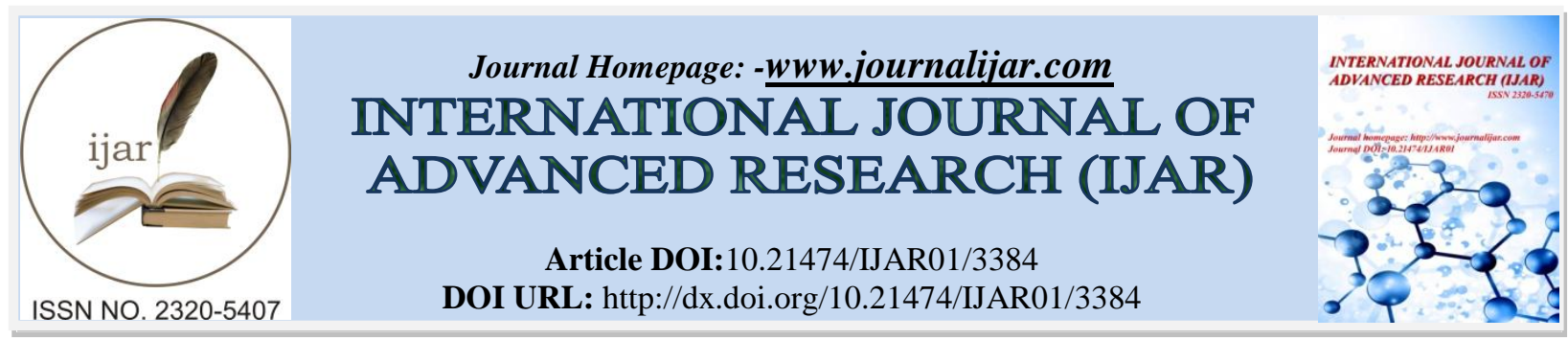

RESEARCH ARTICLE

\title{
CHARACTERISTICS OF UNDER-TRIAGED PATIENTS WHO GET ADMITTED.
}

\author{
Mohammed Alabdrab Alnabi MD ${ }^{1}$, Faisal Ahmad Katbi $\mathrm{MD}^{2}$, Abdullah Ali khunayn $\mathrm{MD}^{2}$, Ghufran \\ Abdullah Alarfaj $\mathbf{M D}^{2}$, Ibrahim Osman $\mathrm{MPH}^{3}$, Asma Ali Almaimoni $\mathrm{MD}^{2}$, Rawan Othman Bamousa $\mathrm{MD}^{4}$, \\ Nora Nasser Aljoaib MD $^{5}$ \\ 1. Maternity \&Children Hospital,Dammam. Saudi Arabia. \\ 2. Imam Abdulrahman Bin Faisal University, Dammam, Saudi Arabia. \\ 3. King FahadSpecialist Hospital,Dammam. Saudi Arabia. \\ 4. King Faisal University, Al-Ahsa. Saudi Arabia. \\ 5. Marefa College for Science \&Technology(MCST), Riyadh. Saudi Arabia.
}

\section{Manuscript Info}

Manuscript History

Received: 12 December 2016

Final Accepted: 16 January 2017

Published: February 2017

Key words:-

under-triage, emergency department, CTAS, pain, cancer.

\section{Abstract}

Background: Triage is a key factor to successfully prioritize patients coming to emergency department to identify life-threatening conditions and allocate resources for patients who need immediate management. Under-triage, defined as inappropriate assignment of a low level of severity during triage, is associated with high levels of substantial mortality. the aim is to identify risk factors \& common diagnoses of under-triaged patients.

Methods: retrospective case-control study based on Canadian Triage \& Acuity Scale (CTAS),

both groups were initially trained CTAS 4 or 5 . The case group includes patients who got admitted and the control group includes patients who were discharged.

Sample of 71 patients was collected over 6 months. demographic characteristics, baseline health status, co-morbid diseases, initial complaint, vital signs, pain score and admission \& discharge diagnoses. Results: Pain played an important role in the triage decision. Pain-free patients were more likely to be under-triaged $(42 \%$ of under-triaged patients versus $0 \%$ of correctly triaged patients).Conversely, cancer patients were more likely to be under-triaged than non-cancer patients, and this was statistically significant $(47 \%$ of under-triaged patients had cancer, versus $20 \%$ of correctly triaged patients, $\mathrm{p} 0.023$ ).

no statistical difference in average age or gender of under-triaged versus correctly triaged patients.

Under-triaged patients were relatively healthier, with less comorbid diseases, although this difference was not statistically significant $(44 \%$ of under-triaged patients were healthy, versus $26 \%$ of correctly triaged patients, $\mathrm{p}$ 0.137).

Conclusion: Factors that ledto under-triage include being cancer patient and pain-free at presentation. We recommend paying attention to those patients during triage. 


\section{Introduction:-}

Triage is the key factor for successful management and prioritization of emergency patients to identify lifethreatening presentations and to allocate resources for patients who need immediate medical management. One of the biggest problems in the triage area is deciding who is truly sick. ${ }^{1}$ Mis-triage (inappropriate triage) can have serious consequences for patients with urgent complaints. ${ }^{2}$ Under-triage, defined as inappropriate assignment of a low level of severity during triage, delays the initiation of treatment and may lead to deterioration of severely ill or injured patients. ${ }^{3}$

Since under-triage is a major concern, A lot of studies have been conducted. Many of them stated that under-triage is associated with high levels of substantial mortality. ${ }^{4-6}$ It is also associated with higher costs because of the use of more interventions and diagnostics. And it is correlated with considerable poor outcomes, longer hospitalization, complications, and morbidity ${ }^{6,7}$

There are many factors that contribute to under-triaging. Some factors are related to nurses and medical staff, such as lack of knowledge, training, and awareness regarding pain perception and management, which represent the main causal factors of under-triage. ${ }^{8}$ Another study found that absence of vital signs measurement, poor recognition of neurological symptoms, and atypical clinical presentations, were associated with under-triage. ${ }^{9}$ For example, normalized vital signs as a reason to forego objective testing for symptomatic patients with a risk factor for PE should not be relied on. ${ }^{10}$ On the other hand, some other factors were more related to patients themselves; such as gender, age, and clinical presentation. A study in Denmark stated that women had a higher risk of being undertriaged than men. Elderly patients were at a significantly higher risk of being under-triaged as well, especially those older than 65 years, and it can have serious clinical consequences. ${ }^{11,12}$

Other studies showed that the common diagnoses of under-triaged patients range from falls from low height ${ }^{11}$ to severe traumatic injuries, like chest, abdomen, head, and pelvis. ${ }^{13,14}$ In one of those studies, traumatic brain injury (TBI) was the most common diagnosis of the under-triaged patients. ${ }^{15} \mathrm{~A}$ study conducted in the United States suggested that some critically ill patients especially those with sepsis were also under-triaged. ${ }^{16}$ In another study, the under-triage group predominantly included elderly males with head and neck injuries, or hemato-oncology diseases. $^{17}$

As the previous studies suggested, under-triaging is associated with serious clinical consequences and poor outcomes. Since it is a major concern, we conducted the study in order to identify patient-related risk factors \& common diagnoses associated with under-triaged patients. We identified under-triage by including patients who were initially assigned a Canadian Triage \& Acuity System (CTAS) triage level of either class 4 or 5, but then got admitted to the inpatient services.

\section{Methodology:-}

A retrospective case-control study at king Fahad specialist hospital (the only cancer center in eastern province). Charts of all admissions originating from the emergency department were screened and reviewed to find out the initial CTAS categories assigned in the period of 6 months. we had 2 categories:

1. Patients admitted from the emergency department but were initially given a low triage priority (CTAS 4 or 5).

2. Patients discharged from the emergency department and were initially given a low triage category (CTAS 4 or 5).

Data of 71 patients were analyzed and demographic characteristics, baseline health status, co-morbid diseases, initial complaints, vital signs, pain score, and final admission \& discharge diagnoses

\section{Results:-}

There was no statistical difference in the average age of under-triaged versus correctly triaged patients (31.6 vs 40.6 years, p 0.104). There was also no statistical difference in patients who were older than 65 years (11\% undertriaged), versus those younger than 65 years (17\% under-triaged, $\mathrm{p} 0.514)$. There was also no statistical difference 
between males \& females, although there was a trend indicating that more females were under-triaged $(61 \%$ of under-triaged patients were female vs $40 \%$ of correctly triaged patients, p 0.098).fig. 1

Under-triaged patients were relatively healthier, with less percentage of comorbid diseases, although this difference was not statistically significant ( $44 \%$ of under-triaged patients were healthy, vs $26 \%$ of correctly triaged patients, $p$ $0.137)$.

Conversely, cancer patients were more likely to be under-triaged than non-cancer patients, and this was statistically significant (47\% of under-triaged patients had cancer, vs $20 \%$ of correctly triaged patients, p 0.023).fig. 2

Pain played an important role in the triage decision. Pain-free patients were more likely to be under-triaged (42\% of under-traigedpatients versus $0 \%$ of correctly triaged patients), whereas patients with a pain score of 6 were more likely to be correctly triaged ( $3 \%$ of under-traiged patients versus $21 \%$ of correctly triaged patients) p 0.013 . fig. 3

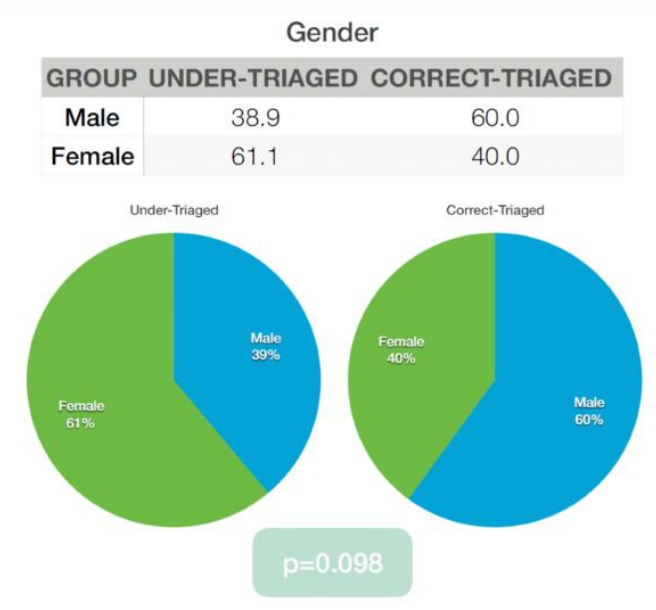

Fig.1:-Effect of gender on patient's triage score.

\begin{tabular}{|c|c|c|}
\multicolumn{3}{|c|}{ Cancer } \\
\hline Group & UNDER-TRIAGED & CORRECT-TRIAGED \\
\hline Yes & 47.2 & 20 \\
\hline
\end{tabular}

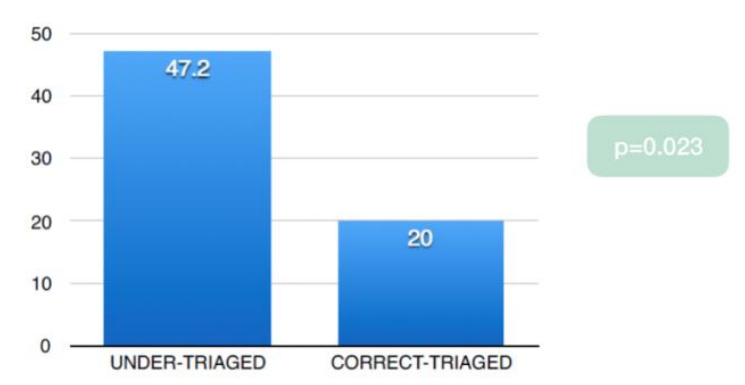

Fig 2:- Comparison between cancer patients who were correctly triaged and those who were under-triaged. 


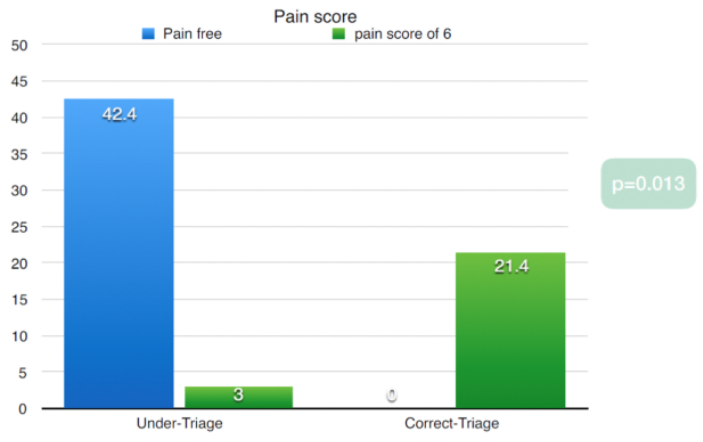

\section{Discussion:-}

Fig. 3:- the pain score as an influencing factor in the process of triaging.

Triage is the key factor for successful management and prioritization of emergency patients to identify lifethreatening presentations and to allocate resources for patients who need immediate medical management. ${ }^{1}$ Undertriage, defined as inappropriate assignment of a low level of severity during triage, delays the initiation of treatment and may lead to deterioration of severely ill or injured patients. ${ }^{3}$

Many factors were studied including the patient's demographics, general condition, co-morbidities and presenting complaint in order to identify factors that led to under-triage. Regarding demographics, the literature states that old age -especially more than 65 years-and female gender are risk factors for under-triaging patients ${ }^{11,12,18}$. In our study no statistical significance was found. However, it showed a trend of under-triaging female. (61\% of under-triaged patients were female Vs $40 \%$ of correctly triaged patients, $\mathrm{p}$ 0.098).

When looking at co-morbidities, cancer patients were more likely to be under-triaged (47\% of under-triaged patients had cancer, vs $20 \%$ of correctly triaged patients p 0.023). it might seem counter-intuitive but This might be attributed to that fact that the study took place in tertiary hospital which is the only cancer center in the Eastern Province. So ER doctors might be more inclined to under-triage those patients as chronic complaint.

Regarding the presenting complaint, Pain played an important role in the triage decision. Pain-free patients were more likely to be under-triaged ( $42 \%$ of under-triaged patients vs $0 \%$ of correctly triaged patients). this seems quite fitting and parallel to the international literature ${ }^{19-21 .}$

We faced some limitations in the sense that the study had a convenience sample, which has resulted in missing statistical significance for some results.

Our definition of under-triage might not be the most optimum definition. We did not have any gold standard triage to compare with.

Study was done in a tertiary cancer \& organ transplant center, and might not be generalized to general hospitals.

\section{Conclusion:-}

We concluded thatFactors contributed to under-triage in our study include being a cancer patient $\&$ being pain-free at presentation. We recommend paying more attention to those patients during triage. 


\section{References:-}

1. Reiter, C. ER nurses learn triage intricacies. Knight Ridder Tribune Business News [Washington] 20 Mar 2006: 1.

2. van der Wulp I1, van Stel HF., Adjusting weighted kappa for severity of mistriage decreases reported reliability of emergency department triage systems: a comparative study. J ClinEpidemiol. 2009 Nov;62(11): 1196-201.

3. Ha SO, Lim KS, Kim W, Oh BJ. Clinical Characteristics and Worsening Prognosis for Undertriage Patients in the Emergency Department: A University Affiliated Hospital Observational Study, . J Korean SocEmerg Med. 2011 Dec;22(6):701-708.

4. Haas B1, Gomez D, et. al, Survival of the fittest: the hidden cost of undertriage of major trauma, J Am Coll Surg. 2010 Dec;211(6):804-11.

5. Buurman BM1, van den Berg W, et. al, Risk for poor outcomes in older patients discharged from an emergency department: feasibility of four screening instruments, Eur J Emerg Med. 2011 Aug;18(4):215-20.

6. N Seiger, M van Veen, E W Steyerberg, M Ruige, A H J van Meurs , H A Moll. Undertriage in the Manchester triage system: an assessment of severity and options for improvement. Arch Dis Child 2011;(96);653-657

7. Staudenmayer KL1, Hsia RY, et. al, Triage of elderly trauma patients: a population-based perspective, J Am Coll Surg. 2013 Oct;217(4):569-76.

8. Kumle B1, Wilke P, et. al, Pain therapy in emergency medicine. Focus on emergency admissions, Anaesthesist. 2013 Nov;62(11):902-8, 910-3.

9. Rutschmann OT1, Chevalley T, et. al, Pitfalls in the emergency department triage of frail elderly patients without specific complaints, Swiss Med Wkly. 2005 Mar 5;135(9-10):145-50.

10. Kline JA1, Corredor DM, et. al, Normalization of vital signs does not reduce the probability of acute pulmonary embolism in symptomatic emergency department patients, AcadEmerg Med. 2012 Jan;19(1):11-7.

11. A K Haug, T Wilson, M S Larsen, et al. Level of undertriage in a well established Trauma Registry in Denmark. InjPrev 2010;(16): A252

12. Chang D, et. al,Undertriage of Elderly Trauma Patients to State-Designated Trauma Centers, Arch Surg. 2008;143(8):776-781.

13. Tazarourte K, Dekadjevi H, Sapir D, et al. Ultrasound and prehospital triage: a tool for limiting the undertriage. J Trauma. 2010;69: 997

14. Cannon CM, Braxton CC, Kling-Smith M, Mahnken JD, Carlton E, Moncure M. Utility of the shock index in predicting mortality in traumatically injured patients. J Trauma. 2009;67:1426 -1430.

15. Xiang H1, Wheeler KK, et. al, Undertriage of major trauma patients in the

16. Page 9 13STUDY \#:US EDs, Am J Emerg Med. 2014 Jun 2. S0735-6757(14)00406-9.

17. Yurkova I1, Wolf L., Under-triage as a significant factor affecting transfer time between the emergency department and the intensive care unit. J EmergNurs. 2011 Sep;37(5):491-6.

18. Ha SO, Lim KS, Kim W, Oh BJ. Clinical Characteristics and Worsening Prognosis for Undertriage Patients in the Emergency Department: A University Affiliated Hospital Observational Study, . J Korean SocEmerg Med. 2011 Dec;22(6):701-708.

19. Kuhn L, Page K, Rolley JX, Worrall-Carter L. Effect of patient sex on triage for ischaemic heart disease and treatment onset times: A retrospective analysis of Australian emergency department data. IntEmergNurs. 2014 Apr;22(2):88-93. doi: 10.1016

20. Vigil J, Coulombe P, Alcock J, Stith SS, Kruger E, Cichowski S. How nurse gender influences patient priority assignments in US emergency departments.pain. 2017 Mar;158(3):377-382

21. Capponi R, Loguercio V, Guerrini S, Beltrami G, Vesprini A, Giostra F. Does the Numeric Rating Scale (NRS) represent the optimal tool for evaluating pain in the triage process of patients presenting to the ED? Results of a muticenter study.

22. Acta Biomed. 2017 Jan 16;87(3):347-352.

23. Rupp T, Delaney KA. Inadequate analgesia in emergency medicine. Ann Emerg Med 2004;43:494-503 